\title{
ATENÇÃo AO SUICÍDIO
}

\section{Attention to suicide}

\section{Berenice Rheinreimer ${ }^{1,2}$, Maurício Kunz ${ }^{1,3}$}

De acordo com a OMS a cada ano ocorrem 1 milhão de mortes causadas por suicídio, número maior que a soma das mortes causadas por guerras e homicídios ${ }^{1}$. Até 2030 , as mortes por suicídio podem chegar a 1 milhão e quinhentos mil, caso sejam mantidos os índices de crescimento atuais ${ }^{2,3}$. Para cada morte, ocorrem de 10 a 40 tentativas de suicídio, ou seja, 10 a 40 milhões de tentativas de suicídio ao ano 4 .

Desde 2005, todas as 10 principais causas de morte nos EUA tiveram taxas decrescentes, sendo o suicídio a única exceção, aumentando de 10,9 suicídios em 2005 para 13 suicídios por 100.000 habitantes em $2013^{5}$. No Brasil, as taxas aumentaram de 4,54 em 2005 para 5,26 em 2013, com incidência bastante diferente entre os estados. O RS apresenta a maior taxa do país: 10,1 suicídios por 100.000 habitantes em 2013, quase o dobro da média nacional ${ }^{6}$.

O suicídio é a terceira causa de morte dos 15 aos 44 anos, causando forte impacto social e financeiro. Em 2008 a Academia Nacional de Ciências dos EUA calculou que a perda de produtividade causada pelo suicídio custe aos EUA 11,8 bilhões de dólares a cada ano ${ }^{2}$. Além disso, especialistas em saúde mental estimam que em cada suicídio, de 4 a 6 familiares são diretamente afetados e necessitarão de suporte psicológico ${ }^{7}$.

Apesar de todo este impacto, por que a prevenção em acidentes de trânsito atrai maior investimento financeiro? O Reino Unido, por exemplo, em 2008-9, gastou 19 milhões de libras em programas de segurança no trânsito, mas apenas 1,5 milhão de libras na prevenção do suicídio'. Nos EUA as mortes causadas por câncer de próstata são em menor número que aquelas causadas por suicídio, e também esta área recebe maiores investimentos ${ }^{8}$. O Instituto Nacional de Saúde Mental Norte Americano investe $31 \%$ de sua verba em pesquisas em neurociências e estudos de comportamento, mas apenas $1,4 \%$ em estudos para a prevenção do suicídio ${ }^{5}$. O assunto tem sido negligenciado não apenas pelos governos, como também pelos especialistas: nos últimos 5 anos, nas 2 maiores revistas americanas de psiquiatria, para cada artigo sobre suicidalidade, havia 6 artigos sobre esquizofrenia, doença com um quarto da incidência do comportamento suicida. Além disto, enquanto os trabalhos sobre esquizofrenia englobavam mecanismos fisiopatológicos, aqueles sobre suicídio se restringiam a registros epidemiológicos ${ }^{1}$.

Mas existem algumas explicações para o pequeno número de pesquisas na área:

- a inexistência de um modelo animal para suicídio .

- a falta de um consenso se as pesquisas devem ser conduzidas apenas com aqueles pacientes que fizeram tentativas de suicídio com franca intenção de morrer ou se devem incluir todos aqueles que apresentarem auto injúria deliberada ${ }^{9}$.

- a conceituação do comportamento suicida como um sintoma ou como uma complicação e não como uma desordem em si ${ }^{1}$.
Clin Biomed Res. 2015;35(3):123-125

1 Programa de Pós-graduação em Psiquiatria e Ciências do Comportamento, Universidade Federal do Rio Grande do Sul (UFRGS). Porto Alegre, RS, Brasil.

2 Hospital Presidente Vargas. Porto Alegre, RS, Brasil.

3 Departamento de Psiquiatria e Medicina Legal, Faculdade de Medicina, Universidade Federal do Rio Grande do Sul (UFRGS). Porto Alegre, RS, Brasil.

Autor correspondente:

Mauricio Kunz

E-mail: maukunz@gmail.com Hospital de Clínicas de Porto Alegre Rua Ramiro Barcelos, 2350.

90035-903, Porto Alegre, RS, Brasil. 
- os gatilhos para o comportamento suicida envolvem não somente a doença mental, mas também dificuldades financeiras e sociais ${ }^{2}$.

- o suicídio ainda é um tabu social e religioso, e em alguns países, é considerado crime e as tentativas de suicídio são passíveis de punição legal ${ }^{1,10}$.

O resultado é que pouco se sabe sobre os mecanismos subjacentes ao comportamento suicida. Mesmo a epidemiologia do suicídio não é totalmente conhecida, com diversas especificidades regionais. Na cidade de São Paulo, um levantamento dos suicídios ocorridos entre 1996 e 2009, mostrou uma diminuição nas taxas dos homens de 65 anos ou mais, e um aumento nos homens entre 25 e 44 anos, sendo que as taxas dos jovens tornaram-se mais elevadas do que as dos idosos . O oposto do que é observado na maioria dos estudos em outros locais, mostrando como o levantamento epidemiológico é fundamental para o desenvolvimento de políticas de prevenção ${ }^{11}$.

Enquanto o suicídio é mais frequente em homens, as tentativas de suicídio são, em sua maioria, praticadas por mulheres jovens, o que mostra uma grande diferença do perfil entre as pessoas que tentam o suicídio e daquelas que dão fim a própria vida $^{2,12}$. Não apenas com relação ao sexo, mas também no que diz respeito à idade, o suicídio e o comportamento suicida apresentam características próprias. Um estudo realizado na Suíça examinou todos os suicídios no país no período de 1998-2007, comparando os métodos usados pelos adultos aos métodos usados pelos jovens com menos de 19 anos. Jogar-se na frente de um trem provocou $20,8 \%$ das mortes dos meninos e $31,8 \%$ das mortes das meninas suíças, quase não ocorrendo entre os adultos. Talvez a dificuldade de acesso a armas de fogo (método mais usados pelos homens suíços) e a medicamentos (método mais usado pelas mulheres suíças) tenha provocado tal diferença ${ }^{13}$. Estes dados mostram que a epidemiologia do comportamento suicida pode variar conforme o sexo, a idade, cultura, região geográfica ou exposição à estressores específicos.

A partir dos dados epidemiológicos podem ser estabelecidas políticas de prevenção ao suicídio. Um programa completo de prevenção abrange a informação da população leiga sobre quais são os sinais de alerta para risco de suicídio e a capacitação de profissionais de saúde da rede básica, já que muitos suicidas buscam atendimento médico nas semanas que antecedem sua morte ${ }^{1}$. Sabe-se que este tipo de treinamento pode reduzir o número de suicídios em $22-73 \%{ }^{2}$. Os programas de prevenção devem ainda investir na facilidade de acesso à atendimento em saúde mental. Outra medida que mostrou a redução do número de suicídios em até $23 \%$ foi a restrição do acesso a meios potencialmente letais, como a diminuição do tamanho das embalagens de Paracetamol, a modificação do gás de cozinha, o uso de catalisadores em automóveis para impedir a intoxicação com monóxido de carbono, a diminuição do acesso a armas de fogo, colocação de grades de proteção para altura em locais públicos, entre outros $^{2,5,8,9}$.

São necessárias ações conjuntas do governo, dos profissionais da saúde e dos pesquisadores para que se alcance uma compreensão mais abrangente do comportamento suicida. Só assim serão desenvolvidas estratégias adequadas de prevenção. Muito ainda pode ser feito.

\section{REFERÊNCIAS}

1. Aleman A, Denys D. A road map for suicide research and prevention. Nature. 2014;509(7501):421-3. http://dx.doi.org/10.1038/509421a. PMid:24860882.

2. Nock MK, Borges G, Bromet EJ, Cha CB, Kessler RC, Lee S. Suicide and suicidal behavior. Epidemiol Rev. 2008;30(1):133-54. http:// dx.doi.org/10.1093/epirev/mxn002. PMid:18653727.

3. Lozano R, Naghavi M, Foreman K, Lim S, Shibuya K, Aboyans V, et al. Global and regional mortality from 235 causes of death for 20 age groups in 1990 and 2010: a systematic analysis for the Global Burden of disease study
2010. Lancet. 2012;380(9859):2095128. http://dx.doi.org/10.1016/S01406736(12)61728-0. PMid:23245604.

4. Williams-Johnson J, Williams E, Gossell-Williams M, Sewell CA, Abel WD, Whitehorne-Smith PA. Suicide attempt by self-poisoning: characteristics of suicide attempters seen at the emergency room at the university hospital of the West Indies. West Indian Med J. 2012;61(5):52631. http://dx.doi.org/10.7727/ wimj.2012.209. PMid:23441377.

5. Swanson JW, Bonnie RJ, Appelbaum PS. Getting serious about reducting suicide. JAMA. 2015. Epub ahead of print. http://dx.doi.org/10.1001/ jama.2015.15566.
6. Brasil. Ministério da Saúde. DATASUS: óbitos por causas externas. Ministério da Saúde; 2014. [citado 2014 Out 01]. Available from: http://tabnet.datasus.gov.br/cgi/tabcgi. exe?sim/cnv/ext10uf.def

7. Park S, Kim CY, Hong JP. Unnatural causes of death and suicide among former adolescent psychiatric patients. J Adolesc Health. 2013;52(2):207-11. http://dx.doi. org/10.1016/j.jadohealth.2012.05.018. PMid:23332486.

8. Suicide watch. [editorial]. Nature. 2014;506(7487):131. http:// dx.doi.org/10.1038/506131a. PMid:24527496. 
9. Pompili M, Vichi M, Leo DD, Pfeffer C, Girardi P. A longitudinal epidemiological comparison of suicide and other causes of death in Italian children and adolescents. Eur Child Adolesc Psychiatry. 2012;21(2):11121. http://dx.doi.org/10.1007/s00787011-0238-5. PMid:22286089.

10. World Health Organization (WHO). Preventing suicides: a global imperative. Luxemburg: WHO; 2014.
11. Bando DH, Brunoni $A R$, Fernandes GF, Bensenor IM, Lotufo PA. Suicide rates and trends in São Paulo,

Brazil, according to gender, age and demographic aspects: a joinpoint regression analysis. Rev Bras Psiquiatr. 2012;34(3):286-93. http:// dx.doi.org/10.1016/j.rbp.2012.02.001. PMid:23429774.

12. Hawton K, Harriss L. How often does deliberate self-harm occur relative to each suicide? A study of variations by gender and age. Suicide Life Threat Behav. 2008;38(6):650-60. http:// dx.doi.org/10.1521/suli.2008.38.6.650. PMid:19152296.

13. Hepp U, Stulz N, Unger-Koppel J, Ajdacic-Gross V. Methods of suicide used by children and adolescents. Child Adolesc Psychiatry. 2012;21:67-73. 